\title{
Transition of Liesegang precipitation systems: simulations with an adaptive grid PDE method *
}

\author{
Paul A. Zegeling ${ }^{\dagger} \quad$ István Lagzi ${ }^{\ddagger} \quad$ Ferenc Izsák ${ }^{\S}$
}

April 1, 2010

\begin{abstract}
The dynamics of the Liesegang type pattern formation is investigated in a centrally symmetric two-dimensional setup. According to the observations in real experiments, the qualitative change of the dynamics is exhibited for slightly different initial conditions. Two kinds of chemical mechanisms are studied; in both cases the pattern formation is described using a phase separation model including the CahnHilliard equations. For the numerical simulations we make use of an adaptive grid PDE method, which successfully deals with the computationally critical cases such as steep gradients in the concentration distribution and investigation of long time behavior. The numerical simulations show a good agreement with the real experiments.
\end{abstract}

*P.A. Zegeling would like to acknowledge the support from the Dutch BSIK-project BRICKS. The research of I. Lagzi and F. Izsák has been supported through the OTKAproject K68253 and the Bolyai Research Fellowship of the Hungarian Academy of Sciences.

${ }^{\dagger}$ Department of Mathematics, Utrecht University, P.O. Box 80010, 3508 TA Utrecht, The Netherlands

${ }_{\ddagger}^{\ddagger}$ Department of Chemical and Biological Engineering, Northwestern University, 2145 Sheridan Rd, Evanston, IL 60208

$\S$ Department of Applied Analysis and Computational Mathematics, Eötvös University, H-1117 Budapest, Pázmány sétány 1/C, Hungary \& Department of Applied Mathematics, University of Twente, P.O. Box 217, 7500 AE Enschede, The Netherlands

ฯCorresponding author. Email: izsakf@cs.elte.hu, Fax: +36-13812158 


\section{Introduction}

It is more than 110 years that Liesegang observed and reported an interesting phenomenon [19]: the precipitate in some simple chemical reactions may not homogeneously distribute. In the typical experimental setup, one chemical reagent is uniformly distributed in a gelled medium (called inner electrolyte), while the other one (called outer electrolyte) diffuses from outside. The initial concentration of the outer (invading) is chosen to be much larger than that of inner one. This condition ensures the higher diffusion flux of the outer electrolyte into the gel. In some circumstances, in the wake of the chemical front some precipitation bands are formed, following each other. In 1D the distances between the bands are determined by the geometrical law [15], see Figure 1. For the description of this phenomenon many models have been proposed such as models based on simple supersaturation [16] or competitive particle growth [12, 7] and models based on phase separation [22, 2, 3]. A general framework for the different models in 1D has been recently published [25].

As the pattern formation in $2 \mathrm{D}$ has recently gained a great interest in the engineering of microsystems [13], a number of experimental studies have been performed. Interestingly, different dynamics have been reported for similar - centrally symmetric - experimental setups: in many cases a regular Liesegang pattern evolved [17], [18], in other experiments only one moving precipitation layer was detected [26], [23].

Our aim is to exhibit and reproduce this phenomenon with numerical simulations and to point out that this can happen using the same material coefficients with a slight modification of initial conditions.

For a successful simulation procedure we have to choose

- an adequate model of the underlying chemical mechanism

- an effective numerical method for solving the PDE for the evolution.

Among the possibilities mentioned above, we have chosen the phase separation model, where the time evolution of the precipitate is described with the Cahn-Hilliard equations, which was originally proposed in [6].

According to this model, the precipitate segregates into the low and high density phases if its local concentration reaches a critical threshold ("spinodal point"). The corresponding fourth order PDE serves as an accurate model: the empirical laws for the Liesegang patterns have been verified by 
numerical simulations [2] in a one dimensional setup. The dynamics driven by the Cahn-Hilliard equations have been analyzed in a series of studies, see e.g. $[1,5,4])$ and is still in the focus of theoretical investigations. Note that we investigate the Cahn-Hilliard equation within a reaction-diffusion system.

In the real applications, the regions which are used as a source of one of the reactants are small compared to the scale of the computational domain. This results in difficulties in the traditional numerical simulations due to the high concentration gradient of the outer (invading) electrolyte and precipitate. On the other hand, frequently, pattern formation phenomena have to be simulated over a relatively long time period. In this way, an overly accurate space discretization or too short time steps can easily result in very time consuming simulations. Therefore, it is important to apply an accurate and fast numerical solver, which successfully deals with the above difficulties. Several techniques can improve the computational procedures such as (i) using appropriate numerical integrators; (ii) using parallel program environment (supercomputer, cluster, GRID systems [20] or video card using specially designed program environment (CUDA) [24]), (iii) using appropriate spatial discretization strategies. Moreover, splitting methods [10] and nonconforming Galerkin methods [11, 28] have been utilized for the numerical approximation. We apply a recent adaptive numerical solver based on a moving grid technique, which deals effectively with all of the abovementioned difficulties.

\section{The model}

The basic chemical reaction which results in the pattern formation phenomenon can be described by the simple equation

$$
A+B \rightarrow C,
$$

where $A$ and $B$ yield the inner and the outer electrolyte, respectively and $C$ denotes the precipitate. For some species, the precipitate $C$ can react with the excess of $A$ (called redissolution) such that the product $S$ (soluble complex) is formed:

$$
A+C \rightarrow S
$$

As an interesting example we mention the reaction, where

$$
A=\mathrm{NH}_{4} \mathrm{OH}, \quad B=\mathrm{Co}^{2+}, \quad C=\mathrm{Co}(\mathrm{OH})_{2}, \quad \text { and } \quad S=\mathrm{Co}\left(\mathrm{NH}_{3}\right)_{6}^{2+}
$$




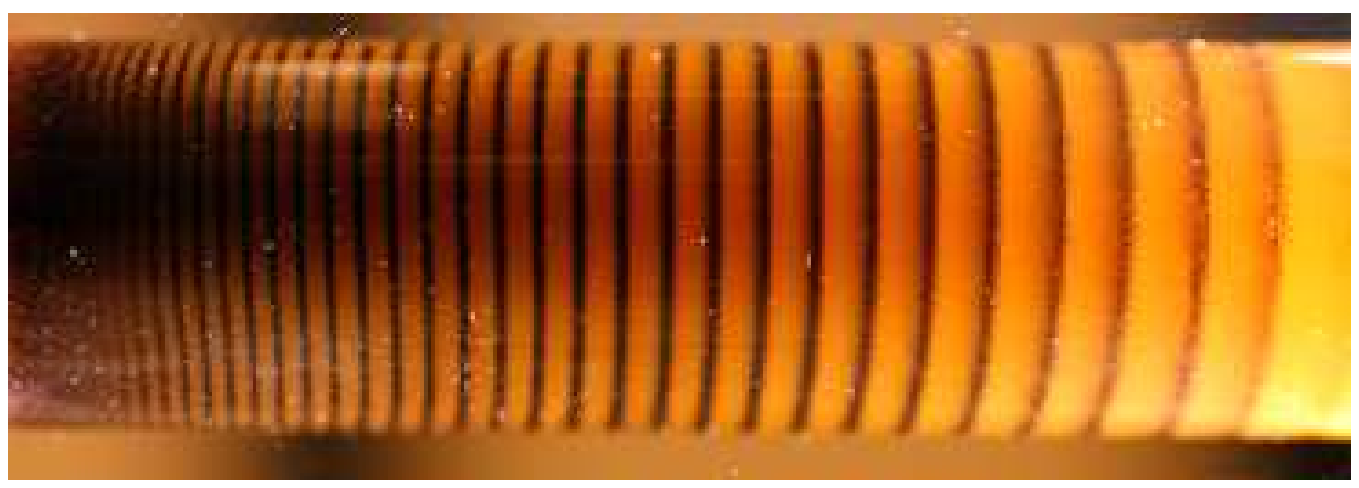

Figure 1: The silver dichromate Liesegang pattern in a test tube. The outer electrolyte invades from the left end of the tube; the dark regions correspond yield the precipitate. The concentration of the inner (potassium dichromate) and outer (silver nitrate) electrolyte are $0.01 \mathrm{~mol} / \mathrm{L}$ and $1.00 \mathrm{~mol} / \mathrm{L}$, respectively.

and, indeed, further species form as well; for a detailed study, see [27]. According to the phase separation model $[22,2]$ :

$$
C \rightarrow C_{\text {high }}+C_{\text {low }},
$$

where $C_{\text {high }}$ and $C_{\text {low }}$ denote the high and the low density fragment of the precipitate, respectively. Practically, pattern formation means in this case that we detect the regions with high density and call them precipitation zones, see Figure 1 . The constants $k_{c}, k_{d}$ and $\lambda$ describe the reaction rate in (1), (2) and (3), respectively.

In mathematical terms, we denote the concentrations of $A, B$ and $C$ with $a, b$ and $c$ respectively, depending on time $t$ and spatial variables $(x, y)$. In all of the consecutive equations, variables in the subscript denote partial derivatives. The above chemical reactions (1-3) correspond to the following system of partial differential equations

$$
\begin{aligned}
a_{t}(t, x, y) & =D_{a} \Delta a(t, x, y)-R_{a} \\
b_{t}(t, x, y) & =D_{b} \Delta b(t, x, y)-R_{b} \\
c_{t}(t, x, y) & =-\lambda \Delta\left(\epsilon c(t, x, y)-\gamma c^{3}(t, x, y)+\sigma \Delta c(t, x, y)\right)+R_{c}
\end{aligned}
$$

which describe the time evolution of $a, b$ and $c$, respectively. $D_{a}$ and $D_{b}$ denote the diffusion coefficients of $A$ and $B$, respectively. The operator $\Delta$ refers 
to the space coordinates $x$ and $y$ and the terms $R_{a}, R_{b}$ and $R_{c}$ correspond to the chemical reactions: either to (1) or to (1-2). The choice of the functions $R_{a}, R_{b}$ and $R_{c}$ will be specified in Section 4 .

The differential operator on the right hand side of (6) describes the dynamics of the phase separation given in (3) by the Cahn-Hilliard equation. For an explanation of the material coefficients $\lambda, \epsilon, \gamma$ and $\sigma$ we refer to [2]. In the numerical simulations, we take all these four constants equal to one. For a discussion on the choice of realistic parameters we refer to [22].

In the mathematical analysis of the Cahn-Hilliard equations on a domain $\Omega$ the Ljapunov functional

$$
\mathcal{E}(c)(t)=\int_{\Omega} \frac{1}{4}\left(c^{2}-1\right)^{2}+\frac{\gamma}{2}|\nabla c|^{2} \mathrm{~d} \Omega
$$

plays a crucial role. From the point of view of physics it can be recognized as free energy. In the absence of source term, i.e. for $R_{c}=0$ in (6), we have $\partial_{t} \mathcal{E}(c)(t)<0$, which determines the evolution of $c$. This case has been extensively studied in $[1,4,5]$. In our case, however, a nontrivial source term $R_{c}$ gives rise to an interesting dynamics.

We simulate the reaction in a radially symmetric setup, in a ring with an inner radius $r_{0}$ and outer radius $R_{0}$, where the unknown concentrations depend only on the distance $r=\sqrt{x^{2}+y^{2}}$ measured from the origin. According to the radial symmetry, $a(t, x, y)=a(t, r, \Phi)=a(t, r)$ gives the spatial dependence of $a$. Using the identity

$$
\Delta a(x, y)=\frac{1}{r}\left[\left[r a_{r}\right]_{r}+\frac{1}{r} a_{\Phi \Phi}\right]=\frac{1}{r} a_{r}+a_{r r},
$$

and a similar, slightly more complicated, one for the operator $\Delta^{2}$ in $\mathrm{PDE}(6)$ we can rewrite the equations in (4-6)

$$
\begin{aligned}
a_{t} & =\frac{D_{a}}{r}\left[a_{r}+r a_{r r}\right]-R_{a} \\
b_{t} & =\frac{D_{b}}{r}\left[b_{r}+r b_{r r}\right]-R_{b} \\
c_{t} & =\left[-\frac{1}{r}-\frac{1}{r^{3}}+\frac{3}{r} c^{2}\right] c_{r}+\left[-1+\frac{1}{r^{2}}\right] c_{r r}+\left[c^{3}\right]_{r r}-\frac{2}{r} c_{r r r}-c_{r r r r}+R_{\varnothing}(9)
\end{aligned}
$$

which are investigated in the interval $\left(r_{0}, R_{0}\right)$ for $0<t<T$ and all of the unknown concentrations depend on $t$ and $r$. 
In the experimental setup, initially, the species $A$ is placed into a small disk $D_{0}$ with radius $r_{0}$, while a homogeneous solution containing the species $B$ is placed into the ring outside of $D_{0}$. The constants -1 and 1 refers to the low and the high density phase of the precipitate, respectively. Indeed, this corresponds to the deviation from the mean concentration, see [22]. Accordingly, we equip (7-9) with the initial conditions

$$
a(0, r)=0, b(0, r)=1 \quad \text { and } \quad c(0, r)=-1 \quad \text { for } r \in\left(r_{0}, R_{0}\right) .
$$

In the real experiments, the continuous inflow of $A$ is ensured at the boundary $\partial D_{0}$ such that here the concentration of $A$ is fixed and is one magnitude larger than that of $B$. It is assumed that the invading species $A$ turns into precipitate such that no outflow occurs at the other boundary of the ring. In practice, the species $B$ is often placed into a gel such that it can not leave this region. The same is valid for the precipitate $C$ such that we apply mixed boundary condition for $A$ and homogeneous Neumann boundary conditions for $B$ and $C$ as follows:

$$
\begin{aligned}
a\left(t, r_{0}\right) & =100, a_{r}\left(t, R_{0}\right)=0 \quad \text { for } t \in(0, T) \\
b_{r}\left(t, r_{0}\right) & =b_{r}\left(t, R_{0}\right)=0 \quad \text { for } t \in(0, T) \\
c_{r}\left(t, r_{0}\right) & =c_{r}\left(t, R_{0}\right)=0 \quad \text { for } t \in(0, T) \\
d_{r}\left(t, r_{0}\right) & =d_{r}\left(t, r_{0}\right)=0 \quad \text { for } t \in(0, T),
\end{aligned}
$$

where $d=c_{r r}$ is an "artificial" PDE variable denoting the second derivative of the precipitate concentration $c(t, r)$. Introduction of the function $d$ makes the PDE system better balanced for the numerical formulation as described in the next section, since we now have four PDEs of second-order, instead of two PDEs of second and one of fourth order. Imposing the last condition means that we take, in fact, the third derivative of $c$ zero at the two boundaries. A natural and consistent initial condition for $d(t, r)$ is: $d(0, r)=0$.

\section{An adaptive moving grid technique}

In the simulations, the concentration gradient is high near those locations, where the high concentration phase can be observed. For an accurate numerical approximation, therefore one has to apply a very fine spatial grid. On the other hand, at those locations, where the concentration of $A$ is low or the low density of $C$ does not result in phase separation, a coarse grid 
would be satisfactory. The locations with high density of $C$ are moving as the precipitation system evolves such that a proper solver should operate with an adaptive moving grid.

\subsection{Transformation of the PDE system to new coordi- nates}

The adaptive moving grid is based on an additional coordinate transformation of the form $r=r(\theta, \rho), t=\theta$, with Jacobian $\mathcal{J}=r_{\rho}$, to obtain the following PDE system in the new coordinates, i.e. $a, b, c$ and $r$ depend now on the variables $\theta$ and $\rho$ :

$$
\begin{aligned}
\mathcal{J} a_{\theta}-a_{\rho} r_{\theta} & =D_{a}\left(\frac{a_{\rho}}{r}+\left(\frac{a_{\rho}}{\mathcal{J}}\right)\right)_{\rho}-\mathcal{J} R_{a} \\
\mathcal{J} b_{\theta}-b_{\rho} r_{\theta} & =D_{b}\left(\frac{b_{\rho}}{r}+\left(\frac{b_{\rho}}{\mathcal{J}}\right)\right)_{\rho}-\mathcal{J} R_{b} \\
\mathcal{J} c_{\theta}-c_{\rho} r_{\theta} & =\left[-\frac{1}{r}-\frac{1}{r^{3}}+3 \frac{c^{2}}{r}\right] c_{\rho}-\mathcal{J} d+\frac{1}{r^{2}} c_{\rho} \\
& +3\left(\frac{c^{2}}{\mathcal{J}} c_{\rho}\right)_{\rho}-\frac{2}{r} d_{\rho}-\left(\frac{d_{\rho}}{\mathcal{J}}\right)_{\rho}+\mathcal{J} R_{c} \\
0 & =\left(\frac{c_{\rho}}{\mathcal{J}}\right)_{\rho}-\mathcal{J} d .
\end{aligned}
$$

The transformation $r(\theta, \rho):[0, T] \times[0,1] \longrightarrow[0, T] \times\left[r_{0}, R_{0}\right]$ is defined in the next section as a solution of a so-called adaptive grid PDE. Note that in the transformed coordinates, in which the PDE solution is expected to behave 'mildly' compared to the original coordinates, a uniform grid with $\Delta \rho=$ constant $=\bar{C}$ is chosen.

\subsection{The adaptive grid PDE method}

To obtain a smooth spatial grid distribution and also smooth grid trajectories in the time direction, we let the adaptive grid transformation $r(\theta, \rho)$ satisfy the following PDE:

$$
\tau_{s}\left[\mathcal{J}_{\theta} \omega\right]_{\rho}+[\mathcal{S}(\mathcal{J}) \omega]_{\rho}=0
$$

with $r(\theta, 0)=r_{0}, r_{\rho}(\theta, 0)=0, r(\theta, 1)=R_{0}, r_{\rho}(\theta, 1)=0$ and initial condition $r(0, \rho)=r_{0}+\left(R_{0}-r_{0}\right) \rho$, i.e., a uniform starting grid. Here, the spatial 
smoothing operator $\mathcal{S}$ is defined by

$$
\mathcal{S}=\mathcal{I}-\sigma(\sigma+1) \bar{C}^{2} \frac{\partial^{2}}{\partial \rho^{2}}
$$

with $\mathcal{I}$ the identity operator and $\sigma>0$ a spatial smoothing constant. Further, $\tau_{s}$ in (15) represents a temporal smoothing constant, which may be taken $\approx 10^{-3} \times$ the 'critical' time-scale in the simulation (small enough to capture rapid solution changes in the time-direction). Finally, the function $\omega$ is a monitor function given by

$$
\omega(\theta, \rho)=\alpha(\theta)+\left|c_{r}\right|, \quad \alpha(\theta)=\int_{r_{0}}^{R_{0}}\left|c_{r}\right| d r .
$$

Remark: For $\tau_{s}=\sigma=0$ (i.e. switching off all smoothing), the adaptive grid PDE (15) reduces to a boundary-value representation of the well-known equidistribution principle

$$
(\mathcal{J} \omega)_{\rho}=0 \Leftrightarrow\left(r_{\rho} \omega\right)_{\rho}=0
$$

with boundary conditions $r(\theta, 0)=r_{0}, r(\theta, 1)=R_{0}$ and initial condition $r(0, \rho)=r_{0}+\left(R_{0}-r_{0}\right) \rho$. A discretization of (17) gives the equidistribution relation

$$
\Delta r_{i} \omega_{i}=\text { constant }
$$

where $\Delta r_{i}$ denotes distance of the $i$ th and $i+1$ th grid points.

It can be proved that for the non-uniform grid arising from the discretization of the adaptive grid PDE (15), the following properties hold:

1) $\mathcal{J}>0, \forall \theta>0 \quad$ (monotonicity of the grid points is preserved)

2) $\mathcal{O}(1)=\frac{\sigma}{\sigma+1} \leq \frac{\Delta r_{i+1}}{\Delta r_{i}} \leq \frac{\sigma+1}{\sigma}=\mathcal{O}(1), \forall i$ and $\forall \theta>0$.

However, taking too large values for $\tau_{s}$ or $\sigma$ results in an unwanted situation namely:

$$
\begin{aligned}
& \text { 3) } \tau_{s} \rightarrow \infty \Rightarrow r_{\theta} \rightarrow 0, \forall \theta>0 \quad \text { (a non-moving grid) } \\
& \text { 4) } \sigma \rightarrow \infty \Rightarrow \mathcal{J} \rightarrow 1, \forall \theta>0 \text { (a uniform grid). }
\end{aligned}
$$

The time-dependent adaptivity parameter $\alpha(\theta)$ serves to smoothly distribute the gridpoints between parts of the domain with high spatial activity $\left(\left|c_{\rho}\right| \gg\right.$ $1)$ and low spatial activity $\left(\left|c_{\rho}\right| \ll 1\right)$. In fact, the choice for $\alpha(\theta)$ in (16) gives 
a grid distribution for which approximately $50 \%$ of the grid points positioned in the region where the first spatial derivative of the precipitate $c(t, r)$ is large and the other half in the remaining part of the domain. Property 2) in (18) with $\sigma=\mathcal{O}(1)$, is of importance to keep the non-uniform grid 'quasi-uniform', i.e. the same formula, $\frac{\Delta r_{i+1}}{\Delta r_{i}}=1+\mathcal{O}\left(\Delta r_{i}\right)$ for each point of time during the simulation. For details on the theoretical background of these properties and the importance of the additional smoothing, we refer to [8, 29] and the references therein. The PDE system in equations (10-14) in combination with (15) is semi-discretized with central finite differences in the $\rho$-coordinate. This results in a large nonlinear system of $5 N$ ordinary differential equations (ODEs), where $N$ denotes the number of spatial gridpoints: $N=\frac{1}{\Delta \rho}$. For the efficient and stable numerical time-integration in the $\theta$-direction BDFmethods are used with variable stepsize and variable order (DASSL, see [21]). In all numerical experiments we took, unless otherwise specified, the following values for the numerical parameters: $\tau_{s}=0.1, \sigma=2, N=400$ and the timetolerance in DASSL $=10^{-6}$.

\section{Simulation results}

We perform the simulations for different values $r_{0}$ of the initial radius, focussing to the case, when this is small compared to the scale of the ring corresponding to an initial point source. This results in large coefficients in (7-9), which can affect the accuracy of the computations. Also, the structure of the formed precipitate can highly depend on the value of $r_{0}[18,23]$.

\subsection{Simulation based on the reaction (1)}

First we investigate the reaction-diffusion system in absence of the redissolution step (2):

$$
R_{a}=R_{b}=R_{c}=k_{c} a b,
$$

with $k_{c}=1$. In the following simulations, computations have been performed on the interval $\left(r_{0}, r_{0}+300\right)$ in the time range $t \in[0,6000]$. The results for various initial radii $r_{0}$ at the final stage $t=6000$ are shown in Figure 2. Comparing the results in Figure 3 one can see that for $r_{0}=0.01$ a single thick precipitation zone evolves. A qualitatively similar evolution can be observed for $r_{0}=0.1$. At the same time, for $r_{0}=1$ and $r_{0}=10$ consecutive precipitation zones appear with an increasing thickness similarly to a regular 

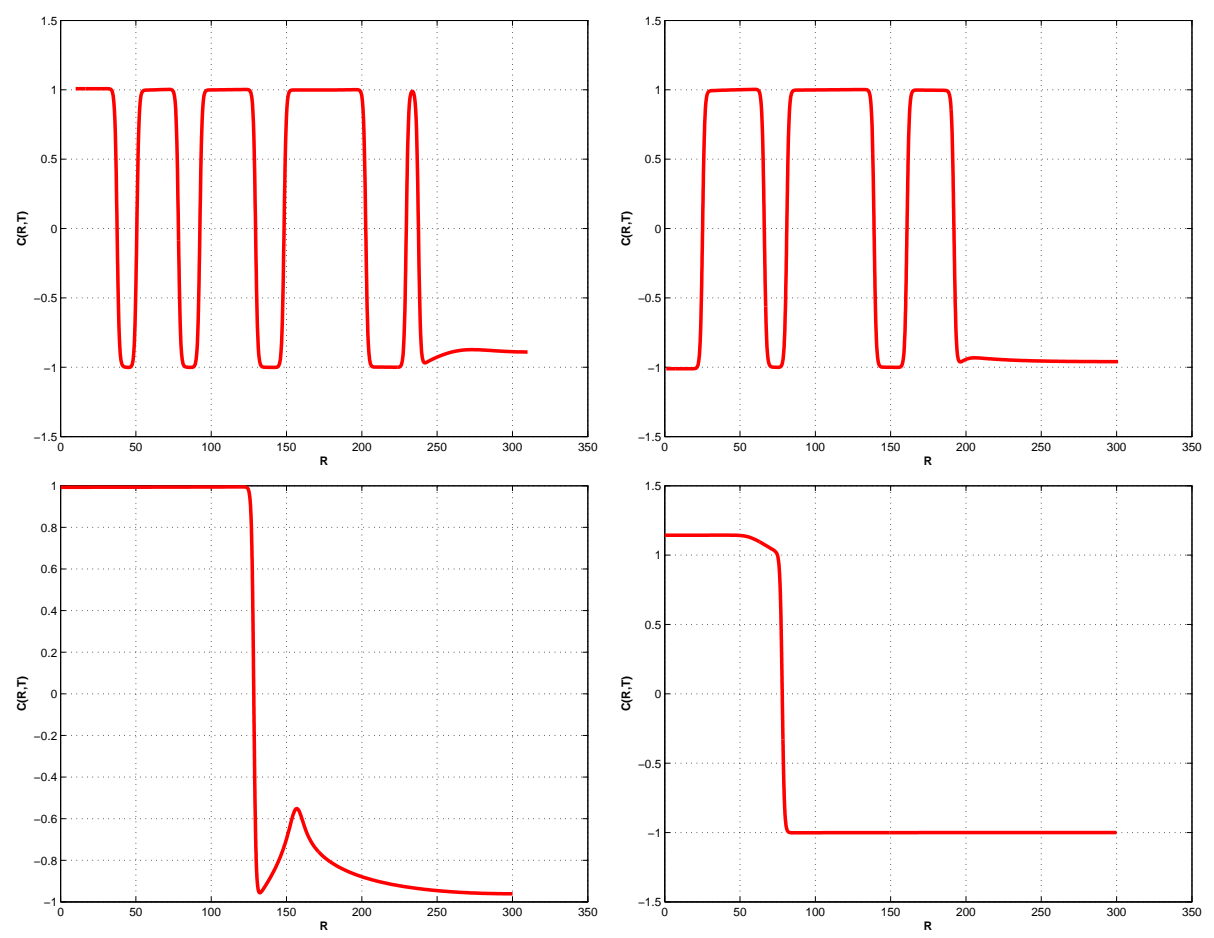

Figure 2: The precipitation pattern in the simulations for $r_{0}=10, r_{0}=$ $1, r_{0}=0.1$ and $r_{0}=0.01$, respectively at $t=6000 . x$ axis : distance from the initial interface of the reactants, $y$ axis: scaled concentration of the precipitate.

one dimensional Liesegang pattern. This corresponds to the experimental observations, see Figure 1.

Note that indeed, we consider a two-dimensional setup such that the classical empirical laws [22], [14], [9] describing the Liesegang patterns are not valid any more.

For a complete description of the reaction, we have also depicted the concentration of the species $A$ and $B$ at the final stage, shown in Figure 3. One can see that the species $B$ is depleted in the region where the phase separation occurred and a relatively small concentration of $A$ can evoke the reaction.

The sharp boundary of the precipitation zone is characterized by the second derivative of the concentration of the precipitate, which are shown 

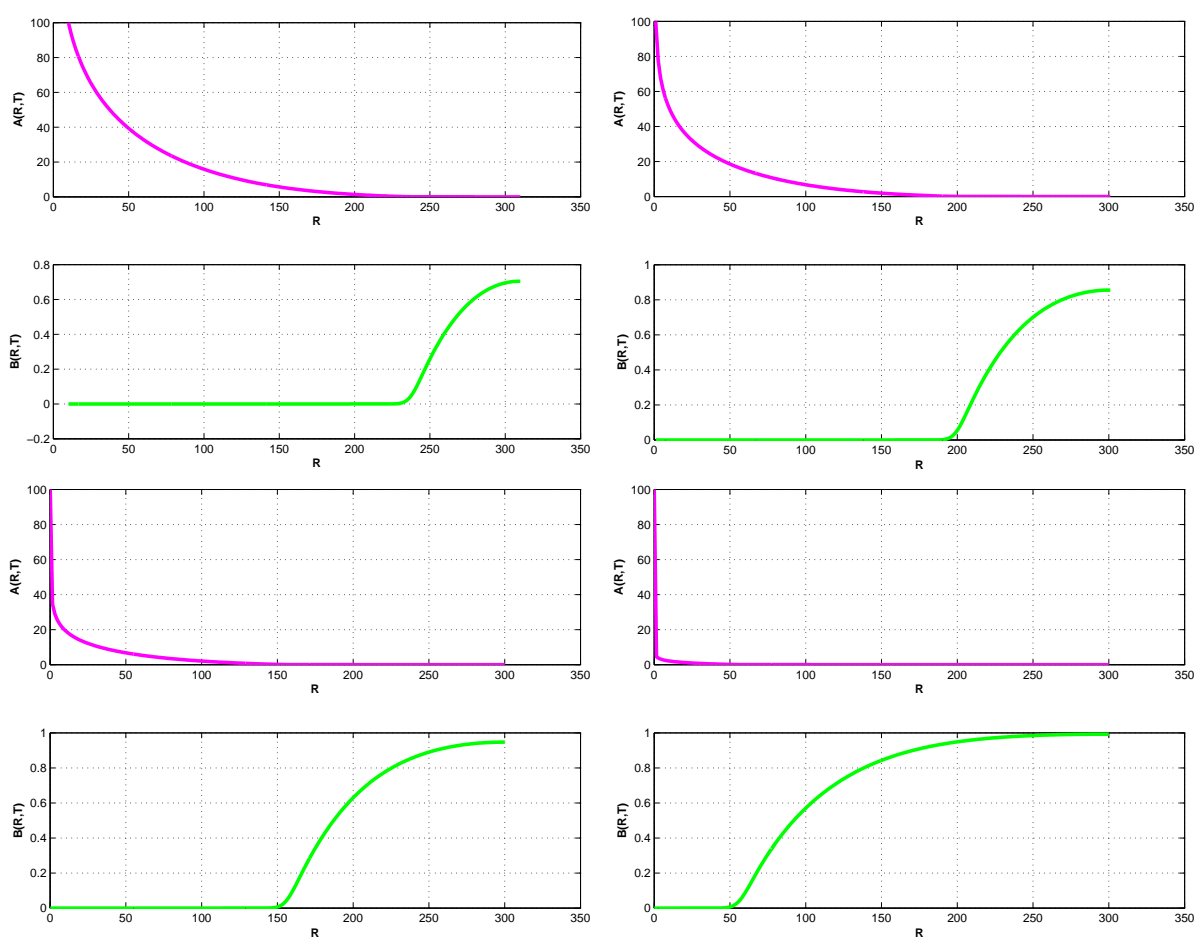

Figure 3: Concentration of the reactants $A$ and $B$ for $r_{0}=10, r_{0}=1$, $r_{0}=0.1$ and $r_{0}=0.01$, respectively at $t=6000 . x$ axis : distance from the initial interface of the reactants, $y$ axis: scaled concentration of the reactants. 

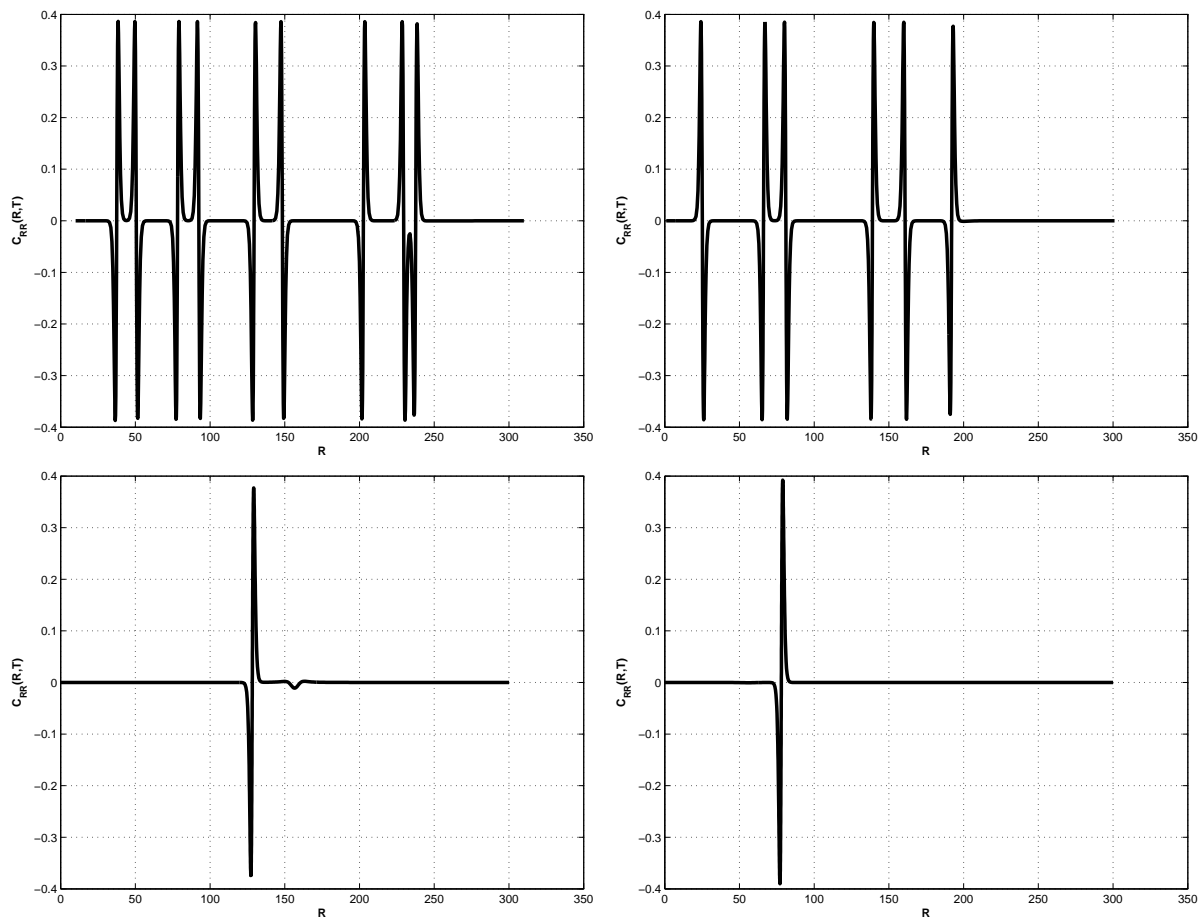

Figure 4: Second derivative $d$ of the concentration function $c$ of the precipitate for $r_{0}=10, r_{0}=1, r_{0}=0.1$ and $r_{0}=0.01$, respectively at $t=6000$. $x$ axis: distance from the initial interface of the reactants, $y$ axis: second derivative of the concentration of $C$.

in Figure 4 for various values of the initial radius $r_{0}$. The time evolution of the precipitate system and the performance of the adaptive moving grid technique can be seen in Figure 5. Here, at time $t$, the horizontal sections indicate the grid points in the tessellation of the interval $\left(r_{0}, r_{0}+300\right)$. As the system evolves, the regions with the fine grid move such that they are always present at the interface of the precipitation zones. These zones become dark in the subfigures. Initially, it takes some time until the grid system is built such that an unstructured dark zone can be observed. During the reaction some precipitation zones can form (see the second subfigure in Figure 5 at time $\approx 4100$ ) and can disappear (see the first and the second subfigure in Figure 5 at time $\approx 5200$ ).

In the adaptation procedure, the grid transformation $r(\theta, \rho)$ is highly influenced by the monitor function $\omega$, which depends on the adaptive pa- 

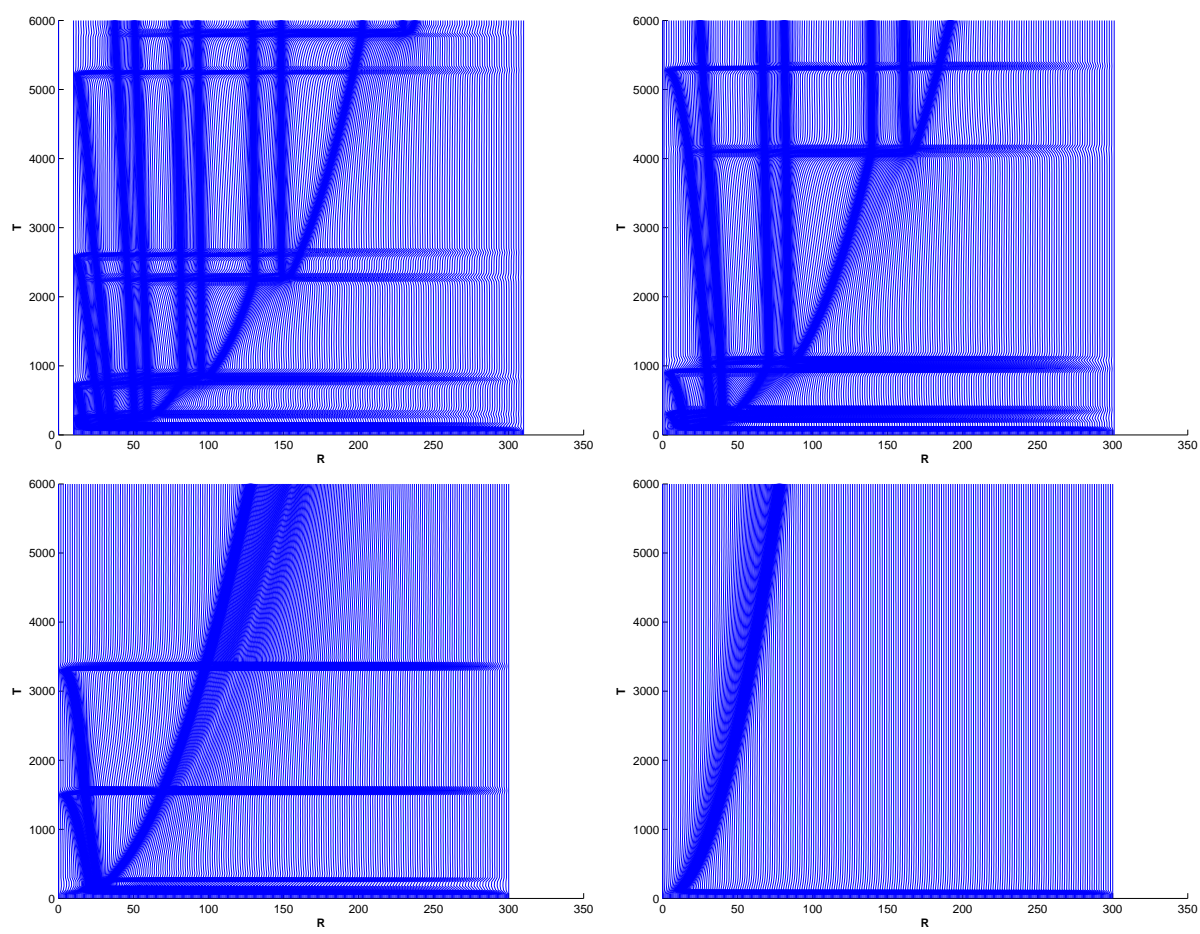

Figure 5: Time variation of the computational grid during the simulation between $t \in[0,6000]$ for $r_{0}=10, r_{0}=1, r_{0}=0.1$ and $r_{0}=0.01$, respectively.

rameter $\alpha(\theta)$. This function is plotted for the different initial radii in Figure 6. Comparing with Figure 5 one can observe that the parameter is increasing when a new precipitation zone forms and decreasing when a zone disappears.

Another point in favor of the adaptive moving grid technique are the relatively low computational costs compared with a uniform (fixed in time) grid. For a comparison in terms of efficiency and accuracy, we refer to the figures in Table 1. There, we have displayed for different numbers of grid points $N$ (both for the adaptive grid and the uniform grid case) not only the CPU time, but also a series of characteristic values of a typical numerical experiment. In the last three columns, respectively, the overshoot (above the value of 1 ), the width of a typical zone and the position on the $r$-axis of this zone are shown. To have a regular pattern structure, we took $r_{0}=200$, $R=1200$ and point of time $t=60000$. We can see that for $N=600$ the adaptive results correspond approximately with the uniform results for $N=3000$. For this case, the adaptive run was almost twice as fast. The 

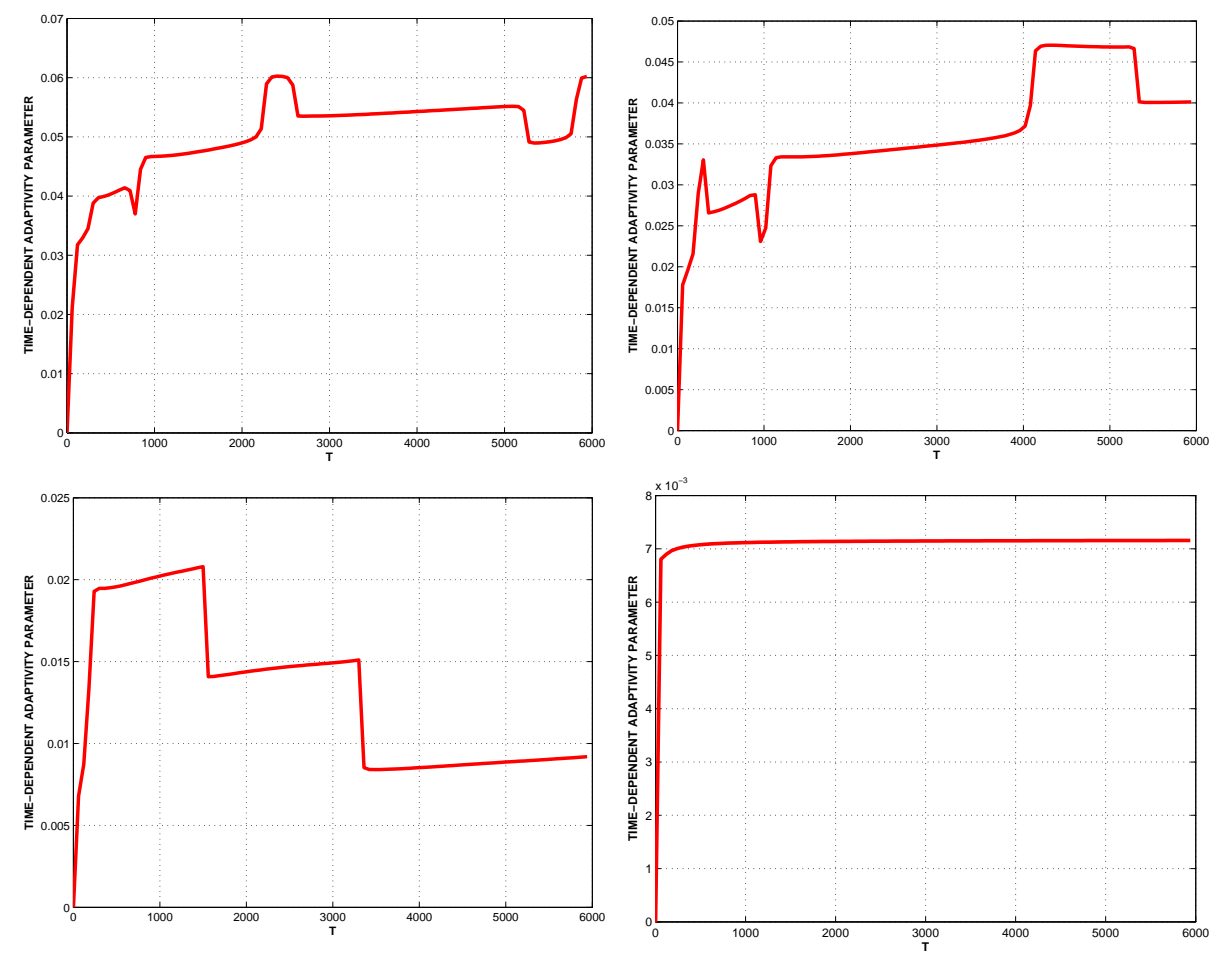

Figure 6: Time variation of the adaptive parameter $\alpha(\theta)$ on the time interval $[0,6000]$ for $r_{0}=10, r_{0}=1, r_{0}=0.1$ and $r_{0}=0.01$, respectively.

uniform grid case with $N=1200$ resulted in very inaccurate solutions which we did not evaluate. These are denoted by the symbol XXX in Table 1 .

\subsection{Modeling of redissolution scenario}

For a more detailed model, we incorporate also reaction step (2) corresponding to the redissolution of the precipitate. It can occur in the excess of the outer electrolyte. Accordingly, the reaction terms in (7-9) are

$$
R_{a}=k_{c} a b-k_{s} a(c+1), \quad R_{b}=k_{c} a b \quad \text { and } \quad R_{c}=k_{c} a b-k_{s} a(c+1) .
$$

In the corresponding simulations (see Figures 7 and 8), we have used $k_{c}=1$ and different parameters $k_{s}=0, k_{s}=0.005, k_{s}=0.0001$, corresponding to the reaction rate in $(2)$. One can observe that the precipitation zones will move during the reaction. To keep track the corresponding dynamics 


\begin{tabular}{|c|c|c|c|c|c|}
\hline \hline$N$ & Method & CPU time & Overshoot & Width & Position \\
\hline \hline 400 & Adaptive & $3 \mathrm{~m} \mathrm{21s}$ & 0.0109 & 16 & 572 \\
\hline 600 & Adaptive & $4 \mathrm{~m} 50 \mathrm{~s}$ & 0.0036 & 24 & 562 \\
\hline 800 & Adaptive & $6 \mathrm{~m} \mathrm{32s}$ & 0.0017 & 22 & 565 \\
\hline 1200 & Uniform & $2 \mathrm{~m} 24 \mathrm{~s}$ & XXX & XXX & XXX \\
\hline 1800 & Uniform & $3 \mathrm{~m} 55 \mathrm{~s}$ & 0.0098 & 112 & 598 \\
\hline 2400 & Uniform & $5 \mathrm{~m} 52 \mathrm{~s}$ & 0.0057 & 118 & 586 \\
\hline 3000 & Uniform & $7 \mathrm{~m} \mathrm{44s}$ & 0.0038 & 20 & 578 \\
\hline 6000 & Uniform & $17 \mathrm{~m} 0 \mathrm{~s}$ & 0.0011 & 21 & 568 \\
\hline
\end{tabular}

Table 1: A comparison of the accuracy and efficiency between adaptive moving grid and uniform grid results for one of the described cases.

precisely, it is essential to apply a moving grid, which becomes dense only at the interface of the low and high phase regions of the precipitate.

\section{Discussion}

In this paper, we exhibited the dependence of the dynamics of Liesegang type precipitation systems on the initial condition: in some cases a regular precipitation system evolves in other cases only one moving precipitation front emerges. This is possible in case of the same material coefficients with an appropriate choice of the radius of the region where one of the reactants is placed. To our best knowledge this transition could not yet been reproduced numerically. The simulations need a special care: steep concentration gradients require locally an accurate spatial discretization locally, and these regions has to be shifted or even cancelled as the system evolves. The method presented here is able to deal with different chemical mechanism as the redissolution scenario and has a clear advantage in terms of the computation costs.

\section{Acknowledgements}

The authors acknowledge the advice and fruitful discussions with Prof. Zoltán Rácz. 

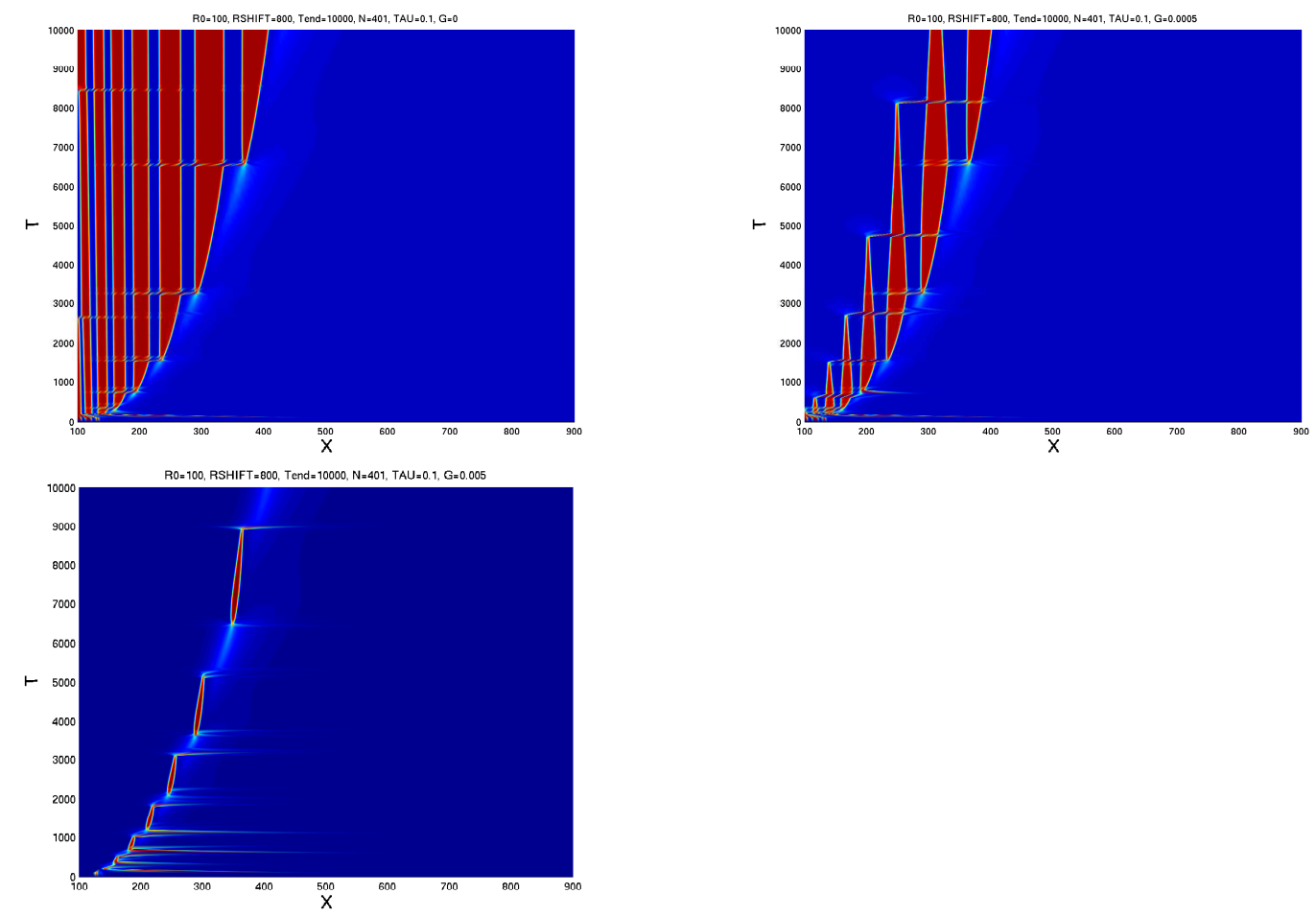

Figure 7: Patterns as a function of space and time for different values of $k_{d}$ in the reaction terms $R_{a}$ and $R_{c}$ in the PDE model for the redissolution. 

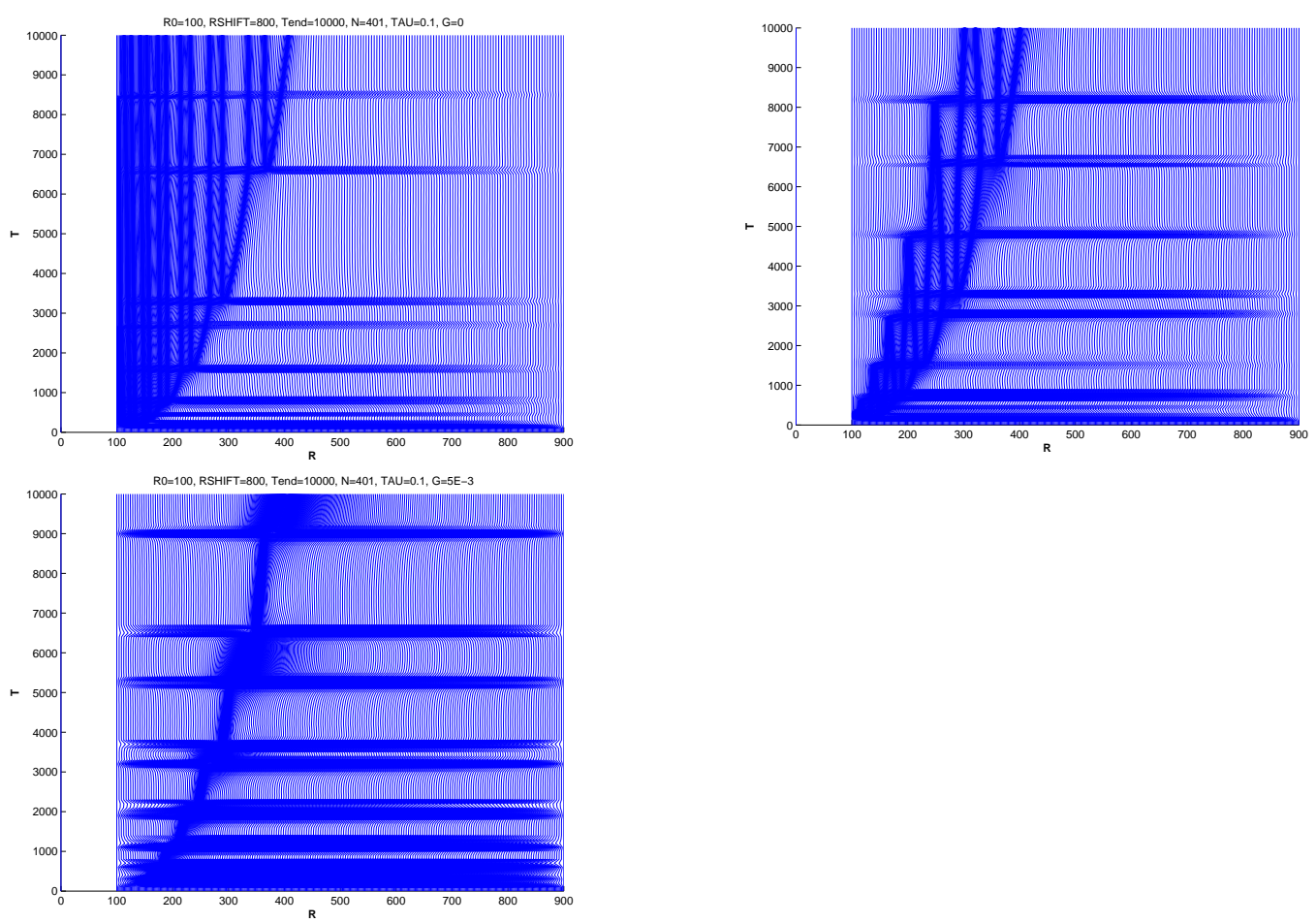

Figure 8: Grid history for different values in case of redissolution. 


\section{References}

[1] N. D. Alikakos, P. W. Bates, G. K. Fusco, Slow motion for the the Cahn-Hilliard equationin one space dimension, J. Differential Equations 90 (1991), 81-135.

[2] T. Antal, M. Droz, J. Magnin, Z. Rácz, Formation of liesegang patterns: A spinodal decomposition scenario, Phys. Rev. Lett. 83 (1999), 28802883.

[3] T. Antal, M. Droz, J. Magnin, A. Pekalski, Z. Rácz, Formation of Liesegang patterns: Simulations using a kinetic Ising model, J. Chem. Phys. 114 (2001), 3770-3775.

[4] P. W. Bates, P. C. Fife, Spectral comparison principles for the CahnHilliard and phase-field equations, and time scales for coarsening, Physica D 43 (1990), 335-348.

[5] P. W. Bates, P. C. Fife, The dynamics of nucleation for the CahnHilliard equation, SIAM J. Appl. Math. 53 (1993), 990-1008.

[6] J. W. Cahn, J. E. Hilliard, Free Energy of a Nonuniform System. I. Interfacial Free Energy, J. Chem. Phys. 28 (1958), 258-267.

[7] M. Chacron, I. L'Heureux, A new model or periodic precipitation incorporating nucleation, growth and ripening, Phys. Lett. A 263 (1999), $70-77$.

[8] A. van Dam, P. A. Zegeling, A robust moving mesh finite volume method applied to $1 D$ hyperbolic conservation laws from magnetohydrodynamics , J. of Comput. Phys. 216 (2006), 526-546.

[9] M. Droz, J. Magnin, M. Zrinyi, Liesegang patterns: Studies on the width law, J. Chem. Phys. 110 (1999), 9618-9622.

[10] C. M. Elliott, D. A. French, A nonconforming finite-element method for the two-dimensional Cahn-Hilliard equation, SIAM J. Numer. Anal. 26 (1989), 884-903.

[11] C. M. Elliott, D. A. French, F. A. Milner, A 2nd-order splitting method for the Cahn-Hilliard equation, Numer. Math. 54 (1989), 575-590. 
[12] R. Feeney, S. L. Schmidt, P. Strickholm, J. Chandam, P. Ortoleva, Periodic precipitation and coarsening waves - Application of the competitive particle growth model, J. Chem. Phys. 78 (1983), 1293-1311.

[13] B. A. Grzybowski, K. J. M. Bishop, C. J. Campbell, M. Fialkowski, S. K. Smoukov, Micro- and nanotechnology via reaction-diffusion, Soft Matter 1 (2005), 114-128.

[14] F. Izsák, I. Lagzi, A new universal law for the Liesegang pattern formation, J. Chem. Phys. 122 (2005), 184707.

[15] K. Jablczynski, Bull. Soc. Chim. France, 33 (1923), 1592-1597.

[16] J.B. Keller, S.I. Rubinow, Recurrent precipitation and Liesegang rings, J. Chem. Phys. 74 (1981), 5000-5007.

[17] H-J. Krug, H. Brandtstädter, Morphological characteristics of Liesegang rings and their simulations, J. Phys. Chem. A 103(39) (1999), 7811-7820.

[18] I. Lagzi, A. Volford, A. Büki, Effect of geometry on the time law of Liesegang patterning, Chem. Phys. Lett. 396 (2004), 97-101.

[19] R.E. Liesegang, Über einige Eigenschaften von Gallerten Naturwiss. Wochenschr. 11 (1896), 353-362.

[20] R. Lovas, P. Kacsuk, I. Lagzi, T. Turányi, Unified development solution for cluster and Grid computing and its application in chemistry, Lect. Notes Comp. Sc. 3044 (2004), 226-235.

[21] L. R. Petzold, A Description of DASSL: A Differential/Algebraic System Solver, in: IMACS Transactions on Scientific Computation, eds.: R.S. Stepleman et al. (North-Holland, Amsterdam, 1993), 65-75.

[22] Z. Rácz, Formation of Liesegang patterns, Physica A 274 (1999), 50-59.

[23] M. Ripszám, Á. Nagy, A. Volford, F. Izsák, I. Lagzi, The Liesegang eyes phenomenon, Chem. Phys. Lett. 414 (2005), 384-388.

[24] A. R. Sanderson, M. D. Meyer, R. M. Kirby, C. R. Johnson, A Framework for Exploring Numerical Solutions of Advection-Reaction-Diffusion Equations Using a GPU-Based Approach, Comput. Visual. Sci. (2008) DOI 10.1007/s00791-008-0086-0 
[25] A. Scheel, Robustness of Liesegang patterns, Nonlinearity 22 (2009), 457483.

[26] R. Sultan, S. Panjarian, Propagating fronts in $2 \mathrm{D} \mathrm{Cr}(\mathrm{OH})_{3}$ precipitate systems in gelled media, Physica D 157 (2001), 241-250.

[27] R. Sultan, Propagating fronts in periodic precipitation systems with redissolution, Phys. Chem. Chem. Phys. 4 (2002), 1253-1261.

[28] G. N. Wells, E. Kuhl, K. Garikipati, A discontinuous Galerkin method for the Cahn-Hilliard equation, J. Comput. Phys. 218 (2006), 860-877.

[29] P. A. Zegeling, Tensor-product adaptive grids based on coordinate transformations, J. Comput. Appl. Math. 166 (2004), 343-360.

[30] P. A. Zegeling, Theory and Application of Adaptive Moving Grid Methods, Adaptive Computations: Theory and Algorithms, ed. T. Tang et al. (Science Press, Beijing, 2007) Chapter 7. 